\title{
COMUNICAÇÃO
}

\section{THE SECOND BURITI WORKSHOP ON TRANSFECTION OF PARASITES}

\author{
Augusto Simões-Barbosa, Nancy R. Sturm and Antônio R.L. Teixeira
}

The development of new technologies for introducing exogenous DNA into parasites has allowed the study of protein function, mechanisms of gene expression and its regulation, drug resistance and designing of new potential chemotherapeutic targets. The use of these modern technologies was the main objective of the Second Buriti Workshop on Transfection of DNA into Parasites, that took place at the Laboratory for Multidisciplinary Research on Chagas Disease, Faculty of Health Sciences of the University of Brasilia, from the $1^{\text {st }}$ to $15^{\text {th }}$ of July, 1995. The Workshop included thirteen investigators from various Brazilian and foreign research institutions, and an equal number of students that were selected from the candidate applicants from São Paulo, Minas Gerais, Goiâs and Pernambuco, and the Federal District.

The theoretical aspects of the Workshop were introduced by Nancy R. Sturm (Department of Microbiology and Immunology, UCLA School of Medicine), who discussed the different methods of transfection along with requirements for electroporation and the various experimental conditions defined in the Workshop protocols. The speaker also discussed some peculiar biological features of kinetoplastid protozoa such as miniexon gene array, polyadenylation and codon usage, and polycistronic sequences and maturation of mRNA.

John M. Kelly (London School of Tropical Medicine) reported on the vector he designed for transfection of Trypanosoma cruzi and Leishmania sp. This vector is based on the plasmid pBluescript and contains two copies

Laboratório Multidisciplinar de Pesquisa em Doença de Chagas. Faculdade de Ciências da Saúde, Universidade de Brasília, Brasilia, DF e Department of Microbiology and Immunology, UCLA School of Medicine, Los Angeles, CA.

Address to: Dr. Antonio R.L. Teixeira, Laboratório Multidisciplinar de Pesquisa em Doença de Chagas, Faculdade de Ciências da Saúde, Universidade de Brasilia. Caixa Postal 04-685, 70919-970 Brasilia, DF, Brasil.

Fax: (061) 273-4645.

Recebido para publicação em 28/02/96. of the GAPDH gene, and the neomycin phosphotransferase gene as a selected marker. The vector characteristics include episomal replication and transformation of parasites resulting in expression ( $1 \%$ to $10 \%$ of total cellular protein) of recombinant proteins. The transfected phenotype can be maintained through the entire $T$. cruzi life cycle in the prolonged absence of drug selection. The p'TEX vector has been used to study the functional aspects of cruzipain and trypanothione reductase, putative targets for chemotherapy ${ }^{35}$.

David A. Campbell (Department of Microbiology and Immunology, UCLA School of Medicine) and David M. Engman (Department of Pathology, Northwestern University School of Medicine, Chicago) discussed several methods for analysing transfected cells using DNA, RNA and protein analysis. After studying several molecular biology methodologies, the students could see the importance of the particular biological features of these organisms. For example, trypanosomatid protein coding genes are expressed as operons with 5' capping, tanssplicing mechanism, and 3 ' polyadenylation. The discussion led to the methods of adding an epitope tag at the carboxyl-terminal end of the recombinant protein and determining its subcellular localization.

Elibio Rech (Cenargen, Brasília, BR) reported on the biolistic principles of transfection, exemplified by the "gene gun". The microbombardment process uses helium gas at high pressure and DNA-coated particles. The particles coated with DNA reach $1500 \mathrm{~km} / \mathrm{h}$ and reach all parts of the cell, including the organelles. This procedure is very usefull in plant cells since their cell walls do not permit the entrance of exogenous DNA by other method of transfection (e.g., lipofection or precipitation with calcium phosphate). The potential for immunization using the gene gun is demonstrated by the successfull vaccination against foot and mouth disease in cattle ${ }^{7}$.

Angela Cruz (Faculdade de Medicina de Ribeirão Preto, SP) spoke about gene targetting 
Comunicação. Simões-Barbosa A, Sturm NR, Teixeira ARL. The Second Buriti Workshop on Transfection of Parasites. Revista da Sociedade Brasileira de Medicina Tropical 29:287-289, mai-jun, 1996.

and genome analysis. Using electroporation techniques she obtained integration and replacement of the DHFR-TS gene of Leishmania major. Success was obtained using a non-virulent cell line but, when she attempeted double knock out of the genes of a virulent cell line, the parasite became either tetraploid or aneoploid to avoid loss of the gene. Among the transfectants, she saw homologous recombination, random integrations and multiple recombination. With the aid of a cosmid library thousands of recombinants were analyzed by an improved methodology $y^{126}$.

Lucille Floeter-Winter (Departamento de Parasitologia, ICB, USP) spoke on the use of transfection to study gene promoters. The analysis of transient transfection of $T$. cruzi and Leisbmania sp. with different constructs containing sequential deletions upstream from the RNA pol I gene, she identified sequences containing promoters of gene expression. The promoter regions are very specific, since they did not function for Cbritidia fasciculata and in some strains of $T$. cruzi.

Bianca Zingales (Instituto de Química, USP) showed that sera from Chagas heart disease patients cross-reacted with the recombinant B13 protein from T. cruzi and with the myosin from cardiac muscle cells. Of interest, these proteins share an epitope of 6 or 7 aminoacids. Further characterization of this epitope is being undertaken.

Experimental work. The second Buriti Workshop concentrated on how to design a successful transfection experiment using electroporation techniques. Seminars and practical exercises demonstrated the steps required for electroporation, such as testing the parameters of the electroporation machine, knowledge of the cell types used in the experiments (drug resistance, growth curve, etc.), and confirmation of results using negative and positive controls.

Characterization of the transfected cells was undertaken using genomic DNA digestion and hybridization by DNA dot and southern blot to demonstrate the presence of the transfected gene of interest and also, the nature of the transfected DNA (episomal or integrated) and an estimative of copy number present in the cell. RNA analysis by northern blot detected mRNA with specific probe thus showing that the exogenous gene was transcribed. The experiments showed how expression of the recombinant protein was regulated. Further, protein analysis of reporter genes showed the vectors used work equally well with Leishmania tarentolae, C. fasciculata and $T$ cruzi. The vectors and the reporter genes, therefore, contain the signals these kinetoplastid protozoann parasites need for expression.

In brief, during two weeks instructors and students that assisted the Workshop designed and performed experiments using transfection procedures. Transfection of parasites has been used tostudy protein function, mechanisms of gene expression and regulation (e.g., promoter sequences), and gene products involved in virulence and drug resistance. The parasites that were electroporated and transfected ( $L$. tarentolae, C. fasciculata and T. cruzi) served as tools for optimization of the working conditions. The experiments led the students to carry on DNA and RNA analyses. For instance, the students demonstrated the presence of the luciferase exogenous gene in transfected T. cruzi cells. Further, recombinant proteins were shown by Western blot and immunocytochemistry assays. The recombinant mHSP70 19E10 and FCB9 9E10 were localized at the kinetoplast and flagellum, respectively, of transfected $T$. cruzi cells, using 9E10 epitope tag antibody.

The Second Buriti Workshop gave the students the opportunity to perform electroporation of parasites and advanced molecular biological, biochemical and immunological techniques for characterization of gene expression in transfected cells. The applications of these techniques will be fundamental in developing new research tools to investigate the kinetoplastid protozoan parasites that cause various endemic diseases in the New World.

\section{ACKNOWLEDGMENTS}

The Organizers of the Second Buriti Workshop acknowledge the financial support from PADCT/CAPES and FAPDF, and technical assistance from Pharmacia Biotech and Sinc do Brasil.

\section{REFERENCES}

1. Cruz A, Beverly SM. Gene replacement in parasite protozoa. Nature 348:171-173, 1990.

2. Cruz A, Coburn CM, Beverly SM. Double targeted gene replacement for creating null mutants. 
Comunicação. Simões-Barbosa A, Sturm NR, Teixeira ARL. The Second Buriti Workshop on Transfection of Parasites. Revista da Sociedade Brasileira de Medicina Tropical 29:287-289, mai-jun, 1996.

Proceedings National Academy Sciences 88:71707174, 1991.

3. Kelly JM, Tomas AM. An approach to functional complementation by introduction of large DNA fragments into Trypanosoma cruzi and Leishmania donovani using a shuttle vector. Molecular Biochemical Parasitology 65:51-62, 1994.

4. Kelly JM, Ward HM, Miles MA, Kendall G. A shuttle vector which facilitates the expression of transfected genes in Trypanosoma cruzi and Leisbmania. Nucleic Acids Research 20:39633969.

5. LeBowitz JH, Coburn CM, McMahon Prat D, Beverly SM. Development of a stable Leishmania expression vector and application to the study of parasite surface antigen genes. Proceedings National Academy Sciences 87:9736-9740, 1990.

6. Pasion SG, Brown GW, Brown LM, Ray DS. Periodic expression of nuclear and mitochondrial DNA replication genes during the trypanosomatid cell cycle. Journal Cell Sciences 107:3515-3520, 1994.

7. Vainstein $\mathrm{MH}$, Alves SA, de Lima BD, Aragão JL, Rech EL. Stable transfection in a flagellate trypanosomatid by microparticle bombardment. Nucleic Acids Research 22:3263-3264, 1994. 